\title{
-Original-
}

\section{Angiogenic Switching in the Alveolar Capillaries in Primary Lung Adenocarcinoma and Squamous Cell Carcinoma}

\author{
Chizuko Morishita ${ }^{1}$, Enjing Jin ${ }^{1}$, Mari Kikuchi ${ }^{1}$, \\ Seiko Egawa', Masakazu Fujiwara', Yoshiharu Ohaki², \\ Mohammad Ghazizadeh ${ }^{1}$, Tamiko Takemura ${ }^{3}$ and Oichi Kawanami ${ }^{1}$ \\ ${ }^{1}$ Department of Molecular Pathology, Institute of Gerontology, Field of Development and Aging Sciences, \\ Graduate School of Medicine, Nippon Medical School \\ ${ }^{2}$ Department of Surgical Pathology, Nippon Medical School Chiba Hokusoh Hospital \\ ${ }^{3}$ Department of Surgical Pathology, Japan Red Cross Center Hospital
}

\begin{abstract}
The status of angiogenic switching was examined in alveolar capillaries of primary lung adenocarcinoma (ADC) from 10 patients and primary squamous cell carcinoma (SCC) from 11 patients, using immunostaining for CD31, thrombomodulin, von Willebrand factor (vWF), collagen types IV and VII, and $\alpha$-smooth muscle actin ( $\alpha$-SMA). We applied the TdT-mediated dUTP nick-end labeling assay and the reverse transcription-polymerase chain reaction for vascular endothelial growth factor (VEGF) and its receptors (VEGFRs). In bronchioloalveolar and papillary subtypes of ADC, the neoplastic cells, replacing the normal alveolar epithelial cells, had spread over alveolar walls and adhered firmly to alveolar interstitium as shown by the development of type IV collagen. Neoplastic cells of SCC were characterized by local proliferation in alveolar sacs without firm attachment to alveolar walls. Tumor lesions of SCC had often developed necrotic foci of various size. In ADC and SCC, alveolar capillary endothelial cells newly obtained reactivity to vWF. Such segments of endothelial cells lost surface thrombomodulin expression. CD31 was consistently expressed in normal and ADC tissues, but each endothelial cell marker was often attenuated or even lost in SCC, suggesting degeneration or necrosis of the alveolar capillaries. The capillary pericytes and interstitial fibroblasts were often hypertrophic and developed $\alpha$-SMA in the cytoplasm in ADC, but they became atrophic in SCC. In ADC, apoptosis occurred in cells of alveolar capillaries more frequently in the peripheral zone than in the deeper zone of the tumor, whereas the frequency was not consistent in SCC. In microdissected alveolar wall tissues, mRNA expression patterns of VEGF isoforms and VEGFRs were similar in both ADC and SCC. In ADC, de novo angiogenic switching took place in cytoplasm as a unit of cells segments in alveolar capillary endothelium. Suppression of angiogenic switching in SCC implies that factors other than VEGF-VEGFR interaction, such as physical contact and compression of tumor cells, might play a critical role in alveolar capillaries.
\end{abstract}

(J Nippon Med Sch 2007; 74: 344-354)

Key words: angiogenesis, alveolar capillary endothelial cell, angiogenic switching, squamous cell carcinoma, adenocarcinoma

Correspondence to Oichi Kawanami, MD, PhD, Director of the Institute of Gerontology, Department of Molecular Pathology, Field of Development of Aging Sciences, Graduate School of Medicine, Nippon Medical School, 1-396 Kosugi-cho Nakahara-ku, Kawasaki, Kanagawa 211-0063, Japan

E-mail:kawanami@nms.ac.jp

Journal Website (http://www.nms.ac.jp/jnms/) 


\section{Introduction}

Tumor growth depends on a local increase in the vasculature, which requires the formation of new capillaries in a process known as angiogenesis. In this process endothelial cells are stimulated to proliferate, sprout, and undergo capillarization in close association with the activation of pericytes and fibroblasts, leading to the formation of basement membrane. The driving force for new vessel formation in tumors is thought to be an increased demand for oxygen and nutrients by neoplastic cells $^{1-4}$. Folkman ${ }^{3}$ has estimated that molecules can diffuse up to approximately $100 \mu \mathrm{m}$ into a tissue from a nearby capillary. Thus, in the absence of local angiogenesis, a tumor cannot grow beyond $2 \mathrm{~mm}^{3,4}$. Once the tumor reaches this limit, hypoxic conditions are present in the center of the neoplastic cell mass and can lead to focal necrosis. Thus, an "angiogenic switching" must occur to promote the growth of new blood vessels ${ }^{2}$.

Vascular endothelial growth factor (VEGF) is a key regulator involved in vascular permeability, endothelial cell proliferation and migration, and vessel maturation. The functions of VEGF are mediated by two tyrosine kinase receptors, VEGFR1 and VEGFR-2 ${ }^{5-10}$. VEGF greatly up-regulates the expression of these receptors on endothelial cells for tumor growth. Using immunofluorescence microscopy, we have previously shown that active angiogenesis occurs at the expanding border of neoplastic cells in primary lung adenocarcinoma $(\mathrm{ADC})^{11-14}$. However, Koukourakis et al..$^{15}$ have shown that the VEGFR-2-activated microvessel density at the invading front of non-small cell lung carcinoma is significantly higher than the microvessel density of adjacent normal tissue. Their immunohistochemical studies have shown that half of the patients expressing higher levels of VEGF had poor vascular density, although the expression of VEGF correlated with the number of new vessels in the remaining patients. This observation suggests that VEGF production by cancer cells does not always involve VEGF/VEGFR-related angiogenic activity and that tumor vessel formation may depend on additional regulators.

In the present study, we focused on the status of angiogenic switching of alveolar capillaries in primary lung $\mathrm{ADC}$ and squamous cell carcinoma (SCC) using immunohistochemical staining for thrombomodulin, von Willebrand factor (vWF), collagen types IV and VII, CD31, and $\alpha$-smooth muscle actin ( $\alpha$-SMA), and the reverse transcriptionpolymerase chain reaction (RT-PCR) for mRNA expressions of VEGF and its receptors. We found that alveolar capillary endothelial cells and the adjacent mesenchymal cells were highly activated for angiogenesis in $\mathrm{ADC}$ but underwent microscopic degeneration/necrosis in SCC; however, mRNA expression patterns of VEGF and VEGFR in SCC remained similar to those in ADC. Thus, it is suggested that the angiogenic switching of alveolar capillaries should be separately taken into consideration in the design of treatments, for $\mathrm{ADC}$ and SCC of primary lung cancer.

\section{Materials and Methods}

\section{Tissues}

Fresh specimens of lung tissue were obtained at lobectomy from 10 patients (6 men and 4 women; average age, 64.2 years; average smoking history, 9.6 pack-years) with solitary primary ADC and from 11 patients (10 men and 1woman; average age, 67.2 years; average smoking history, 47.8 pack-years) with solitary primary SCC. Histologically normal control lung tissues were taken in areas distant from the isolated nodules of 7 patients. This study was approved by the ethics committee associations of the Japan Red Cross Center Hospital and of Nippon Medical School. The primary lung ADC included two histologic types, according to the World Health Organization critera, bronchioloalveolar carcinoma and papillary ADC. The SCC included 3 poorly, 4 moderately, and 4 keratinizing well-differentiated types. Tumor tissue samples were cut into small pieces for cryosectioning either without fixation or with brief fixation in $4 \%$ buffered paraformaldehyde for immunohistochemical or immunofluorescent staining. In addition, some tissues were fixed in $10 \%$ buffered formalin, embedded in paraffin, and cut into 
4- $\mu \mathrm{m}$ sections for immunohistochemical staining, hematoxylin and eosin (H\&E) staining, and TdTmediated dUTP nick-end labeling (TUNEL) analysis. For reverse transcription-polymerase chain reaction (RT-PCR), fresh lung specimens were obtained at lobectomy from 4 patients with ADC (3 men and 1 women; average age, 66.0 years; average smoking history, 13.5 pack-years), and 4 patients with SCC (3 men and 1 woman; average age, 65.0 years; average smoking history, 45.0 pack-years). Histologically normal lung tissues were taken at distant areas from 2 patients with ADC ( 2 men; average age, 71.5 years; average smoking history, 15.0 pack-years) and 2 patients with SCC (2 men; average age, 76.0 years; average smoking history, 15.0 pack-years). Unfixed frozen sections were cut with a razor blade under Clinicut (Bright, Huntingdon, England) and they were further microdissected in the same way as described previously for RT-PCR analysis ${ }^{11,12,14}$.

\section{Immunoperoxidase Staining}

Deparaffinized tissue sections were stained using the streptavidin-biotin complex immunoperoxidase $\operatorname{method}^{11-13}$. For collagen type IV staining, sections were treated with $0.05 \%$ Protease Type VIII (SigmaAldrich Corp. St. Louis, MO, USA) for $15 \mathrm{~min}$ at room temperature, and for $\alpha$-SMA and CD31 staining, sections were boiled in $10 \mathrm{mM}$ citrate buffer ( $\mathrm{pH}$ 6.0) for $10 \mathrm{~min}$. Next, the samples were treated with $0.3 \%$ hydrogen peroxide in methanol for $15 \mathrm{~min}$ at $20^{\circ} \mathrm{C}$ to eliminate endogenous peroxidase activity, followed by $10 \mathrm{~min}$ with $10 \%$ normal rabbit serum to prevent nonspecific antibody binding. Samples were incubated overnight at $4^{\circ} \mathrm{C}$ with monoclonal antibodies against collagen type IV (1 : 100), $\alpha$-SMA (1 : 25), or CD31 (1 : 40)(DAKO, Carpinteria, CA, USA). Finally, immunoreactive protein was detected with 3,3'-diaminobenzidine (DAB), and the sections were counterstained with hematoxylin. Control sections were stained with corresponding amounts of mouse IgG (DAKO) in place of the primary antibodies. The controls consistently gave negative results, although falsepositive staining was observed infrequently in keratinizing cancer cells in SCC. Sections with other false-positive staining were discarded.
Immunohistochemical and Dual Immunofluorescent Staining of Frozen Sections

Tissues that had been briefly fixed in paraformaldehyde were embedded in optimum cutting temperature compound, snap frozen in acetone-dry ice, and stored at $-80^{\circ} \mathrm{C}$ until sectioning. An indirect immunofluorescence method was used to stain 4 - to $30-\mu \mathrm{m}$-thick sections, as described previously $^{12,16}$. Briefly, for type VII collagen staining, the sections were incubated overnight with a $1: 100$ dilution of a mouse monoclonal IgG antibody against type VII collagen (LH7 : 2, Cymbus Bioscience Ltd, Southampton, UK) as the primary antibody. The primary antibody against type VII collagen was reacted with 3,3'-DAB as described above.

For dual immunofluorescent staining as reported previously $^{11-14}$, the sections were incubated overnight at $4{ }^{\circ} \mathrm{C}$ with a combination of a mouse monoclonal IgG antibody against thrombomodulin ( 1 : 50; DAKO) and a rabbit polyclonal antibody against vWF (1 : 400; DAKO). After being washed several times, the sections were incubated for $40 \mathrm{~min}$ at room temperature with a mixture of $1: 100$ dilution Texas red-labeled horse anti-mouse and $1: 100$ fluorescein isothiocyanate-labeled goat anti-rabbit IgG (Vector Laboratories, Burlingame, CA, USA). Nuclear counterstaining was accomplished by incubating the sections for $15 \mathrm{~min}$ at room temperature with $0.01 \%$ TOTO-3 iodide (Molecular Probes, Eugene, OR, USA). These sections were then nuclearcounterstained with $0.01 \%$ 4',6-diamidino-2phenylindole (DAPI; Sigma Chemical Co., St Louis, MO, USA). The sections were examined with a confocal microscope (model TCS4DD/DMIRBE; Leica, Heidelberg, Germany) equipped with argon and argon-krypton laser sources. In the resulting preparations, green fluorescence represented vWF, red represented thrombomodulin, and the nuclei appeared blue.

For both immunohistochemistry and immunofluorescence staining, control preparations consisted of: 1) omission of the primary antibody from the staining procedure; and 2) substitution of corresponding amounts of normal immunoglobulin for the primary antibody. The controls consistently gave negative results. 


\section{TUNEL Assay}

Normal, ADC, and SCC tissues were evaluated using the TUNEL assay to identify endothelial cells undergoing apoptosis. Paraffin sections were stained with the TUNEL method using an In Situ Cell Death Detection Kit, POD (Roche, Mannheim, Germany) according to the manufacturer's instructions. The sections were also stained with methyl green. Forty high-power $(\times 400)$ fields of each section were randomly selected at light microscopy, and the number of TUNEL-positive endothelial cells was calculated.

\section{RT-PCR}

RT-PCR was carried out according to our previously published methods ${ }^{12}$. Briefly, total RNA was extracted using an RNeasy Mini Kit (Qiagen, Hilden, Germany). The RNA was then reversetranscribed into cDNA using an oligo (dT) primer and SuperScript ${ }^{\mathrm{TM}}$ II Reverse Transcriptase (Invitrogen, Carlsbad, CA, USA) at $42^{\circ} \mathrm{C}$ for $1 \mathrm{~h}$. RTPCR was carried out as described previously for VEGF, VEGFR-1, VEGFR-2, and glyceraldehyde 3phosphate dehydrogenase (GAPDH) (internal loading control) using $1 \mu \mathrm{L}$ of the cDNA products and a Expand High Fidelity PCR System (Roche) according to the manufacturer's instructions. Following denaturation at $94^{\circ} \mathrm{C}$ for $10 \mathrm{~min}, \mathrm{PCR}$ was performed for VEGF using primers 5GCTACTGCCATCCAATCGAGACC-3' (sense) and 5'-GT T TCT GGA T T A A GGACTGT TC TGTCG-3' (antisense) for 30 cycles of $1 \mathrm{~min}$ at $94^{\circ} \mathrm{C}, 1 \mathrm{~min}$ at $62^{\circ} \mathrm{C}$, and $2 \mathrm{~min}$ at $72^{\circ} \mathrm{C}$. The expected PCR products were 440, 572, 644, and 695 bp for the 121-, 165-, 189-, and 206-amino acid isoforms of VEGF, respectively. For VEGFR-2, PCR was performed using primers 5'CATCACATCCACTGGTATTGGC-3' (sense) and 5' GGCCAAGCTTGTACCATGTGAG-3' (antisense) for 30 cycles of $45 \mathrm{~s}$ at $94^{\circ} \mathrm{C}, 45 \mathrm{~s}$ at $56^{\circ} \mathrm{C}$, and 2 min at $72^{\circ} \mathrm{C}$. The expected PCR product for VEGFR-2 was 405 bp. PCR was performed for VEGFR-1 using primers 5'-GGCCAAGCTTGTACCATGTGAG-3' (sense) and 5'-GAGGATTTCTTCCCCTGTGTA-3' (antisense) for 30 cycles of $45 \mathrm{~s}$ at $94^{\circ} \mathrm{C}, 45 \mathrm{~s}$ at $60^{\circ} \mathrm{C}$, and $1 \mathrm{~min}$ at $72^{\circ} \mathrm{C}$. The expected PCR product for VEGFR-1 was $513 \mathrm{bp}$. PCR was performed for GAPDH using primers 5'-ACCACAGTCCATGCCATCAC-3' (sense) and 5'-TCCACCACCCTGTTGCTGTA-3' (antisense) for 30 cycles of $30 \mathrm{~s}$ at $94^{\circ} \mathrm{C}, 30 \mathrm{~s}$ at $60^{\circ} \mathrm{C}$, and $1 \mathrm{~min}$ at $72^{\circ} \mathrm{C}$. The expected PCR product for GAPDH was 450 bp. The PCR products were separated with electrophoresis on $2 \%$ agarose gels and visualized with ethidium bromide staining. Semiquantitative measurements of VEGF and VEGFR mRNA isoforms were made by comparing the RT-PCR results with standard curves constructed for VEGF, VEGFR and glyceraldehyde 3-phosphate dehydrogenase. Densitometric analyses were carried out using a Molecular Imager FX and the Quantity One software package (Bio-Rad, Hercules, CA, USA).

\section{Statistical Analysis}

All experiments were repeated three times. Data are presented as means \pm SD. Statistical comparisons were made using Student's $t$-test for unpaired observations (two-tailed), and differences were considered significant when $P$ was less than 0.05 .

\section{Results}

In the present study, we focused on the border area between normal and neoplastic cells in primary $\mathrm{ADC}$ and SCC in the lung. Each of the 10 patients with ADC and the 11 with SCC, showed common histological characteristics in the angiogenic behavior of alveolar capillaries. At the expanding border of primary ADC, the wall surfaces were covered by a single layer of neoplastic cells in bronchioloalveolar carcinoma (Fig. 1-a, upper half) and in papillary ADC. The alveolar wall interstitium often became thick due to collagen deposition. The amount of collagenous matrix tended to increase near the center of the original mass in bronchioloalveolar carcinoma. In papillary ADC, however, most papillary stalks of the alveolar walls expanding in alveolar sacs remained thin, although a new capillary meshwork developed inside the alveolar walls. The tumor cells replaced the normal alveolar epithelial cells and were firmly attached to the alveolar interstitium.

Histological examination showed that SCC was 

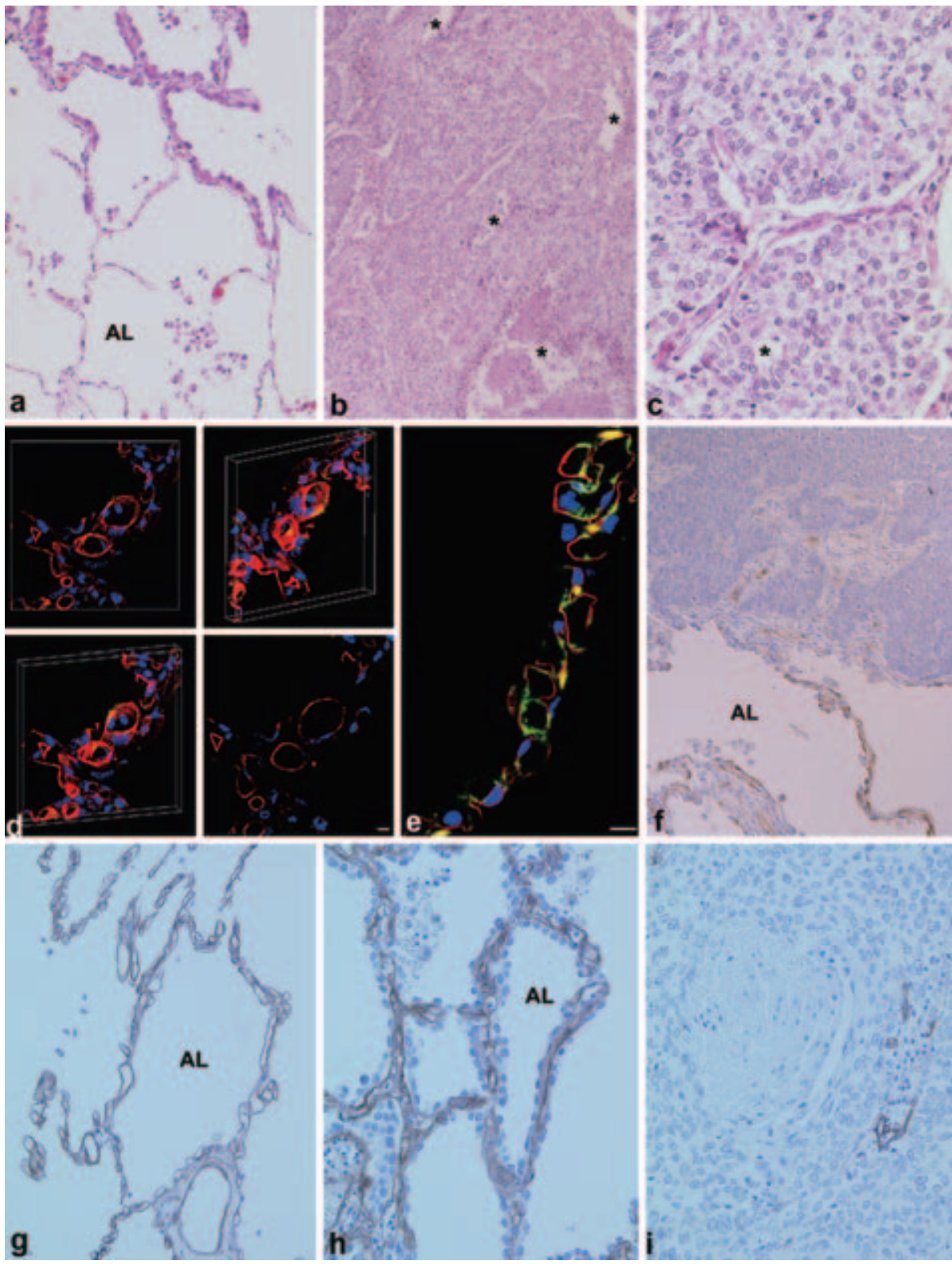

Fig. 1 H\&E staining for ADC (a) and SCC (b and c). Abbreviation. AL, alveolus

a) At the expanding border of primary $\mathrm{ADC}$, at sites where the wall surfaces were covered by a single layer of neoplastic cells in bronchioloalveolar carcinoma, the alveolar wall interstitium often became thick due to collagen deposition (upper half). (original magnification $\times 200$ )

b) In SCC, most neoplastic cells grew into the lumen, and the top surfaces of the tumor were left free from the adjacent alveolar walls. Necrotic foci of cancer cells (asterisks) were also found scattered in tumor lesions in nonkeratinizing types of SCC. Adjacent alveolar walls in the lesions often showed degeneration. $(\times 100)$

c) Alveolar capillaries were irregularly dilated like sinusoids and the nuclei of endothelial cells frequently became pyknotic. $(\times 400)$ Remaining type II alveolar epithelial cells proliferated like a glandular arrangement (asterisk) in occasional areas.

d) In the normal lung, the inner surface of capillaries was covered by a thin sheet of endothelial cells that expressed the anticoagulant thrombomodulin along the cell surfaces. It was obvious that normal alveolar capillaries did not contain cytoplasmic vWF, as shown by a lack of green staining. Bar: $5 \mu \mathrm{m}$

e) In alveolar capillaries located at an immediate distance of tumor invasion in ADC, thrombomodulin expression was often absent in limited segments of endothelial cells. Instead, occasional segments showed irregular amounts of vWF along the capillary loops.

f) At the normal borders of SCC, some capillary endothelial cells showed an occasional loss of thrombomodulin expression, whereas they showed a complete absence of thrombomodulin expression in deeper areas of the tumors in both keratinizing and nonkeratinizing types of SCC. $(\times 100)$

g) Type IV basement membrane collagen was localized along the capillary conduit and subjacent to alveolar epithelial cells in normal lungs. $(\times 200)$

h) In ADC, neoplastic cells developed more distinct basement membranes than did normal cells, and they often formed multiple layers in the alveolar walls. (Immunoperoxidase reaction, $\times 200$ )

i) Basement membranes in proper alveolar structures located deep in the SCC were hardly discernible. (Immunoperoxidase reaction, $\times 200$ ) 
characterized by the proliferation of neoplastic cells that tended to fill the alveolar sacs through an initial invasion site at the alveolar wall. Tumor cells expanded locally and invaded the adjacent normal lung fields. Keratinizing neoplastic cells often developed cancer foci containing areas of central necrosis of various size (Fig. 1-b). The top surfaces of the tumor were left free from the adjacent alveolar walls, whereas most neoplastic cells grew into the lumen as a mass. Thus, the remaining type II alveolar epithelial cells showed a reactive proliferation and occasionally formed a glandular structure in cancer nests (Fig. 1-b, c). Necrotic foci of cancer cells were found scattered in tumor lesions in the nonkeratinizing type of SCC as well. Alveolar capillaries in such lesions were irregularly dilated like sinusoids (Fig. 1-b, c). The nuclei of endothelial cells were frequently pyknotic (Fig. 1-c), and capillary pericytes were highly attenuated in the edematous interstitium. The alveolar interstitium occasionally contained an increased number of interstitial fibroblasts showing focal deposition of collagen matrix, similar to granulation tissue. These changes occurred at varying frequencies on the borders facing the normal alveolar structures in each patient with SCC.

\section{Immunohistochemical and Immunofluorescent Staining}

In the normal lung, alveolar capillaries formed a complicated meshwork of tubular structures in 3dimensional images. The inner surface of capillaries was covered by a thin sheet of endothelial cells that expressed the anticoagulant thrombomodulin (Fig. 1d). Despite consistent expression of $\mathrm{vWF}$ in most vascular endothelial cells, it was obvious that alveolar capillaries did not contain cytoplasmic vWF, as shown by a lack of green staining. However, alveolar capillaries immediately adjacent to areas of tumor invasion in ADC lost thrombomodulin expression in limited segments of endothelial cells. Instead, occasional segments developed irregular amounts of $\mathrm{vWF}$ in the cytoplasm along the capillary loops (Fig. 1-e). In the capillary endothelial cells deep in ADC lesions, vWF and CD31 were consistently expressed in the cytoplasm, whereas thrombomodulin became negative. At the normal borders of SCC, capillary endothelial cells often showed an occasional loss of thrombomodulin expression, but completely lost thrombomodulin expression in deeper areas of tumors in both keratinizing and nonkeratinizing types of SCC (Fig. 1-f). Neoplastic cells in differentiated SCC also showed staining for thrombomodulin in the cytoplasm, although the neoplastic cells were easily distinguishable from capillary endothelium.

Immunohistochemical analysis showed that type IV basement membrane collagen was localized subjacent to alveolar epithelial cells and along the capillary conduit in the normal lungs similarly to the reaction in CD31 (Fig. 1-g). In ADC, neoplastic cells developed more distinct basement membranes than did normal cells, and they often formed multiple layers along the alveolar walls (Fig. 1-h). In most areas near the invasive tip of SCC, type IV collagen was easily detected. In contrast, type IV collagen became indistinct among neoplastic cells, Fragments of basement membranes remained along the proper alveolar structures (Fig. 1-i). Weakly positive or faint shadows of thrombomodulin, vWF, and CD31 (arrow in Fig. 2-a) were observed along preexisting capillary endothelial cells in the alveolar walls deep in SCC.

Although type VII collagen is known to be localized subjacent to bronchial epithelial cells and glandular and duct epithelial cells in the airways ${ }^{16}$, neoplastic cells of ADC or SCC, did not show any staining for type VII collagen along the basal layer of the alveolar walls, but the patchy remnants of preexisting bronchial basement membrane were found along the airways (Fig. 2-b). Pericytes and fibroblasts/myofibroblasts in ADC often had extended cytoplasmic processes containing microfilaments reactive to $\alpha$-SMA. The nuclei of these cells were often hypertrophic and contained relatively conspicuous nucleoli (Fig. 2-c, arrows). In contrast, the cytoplasm of capillary pericytes (Fig. 2-d, arrow) was flat and attenuated in the alveolar walls in SCC lesions, and $\alpha$-SMA was not consistently developed and was often absent in alveolar walls. Capillaries showed irregular dilatation, and the cytoplasm of endothelial cells was 

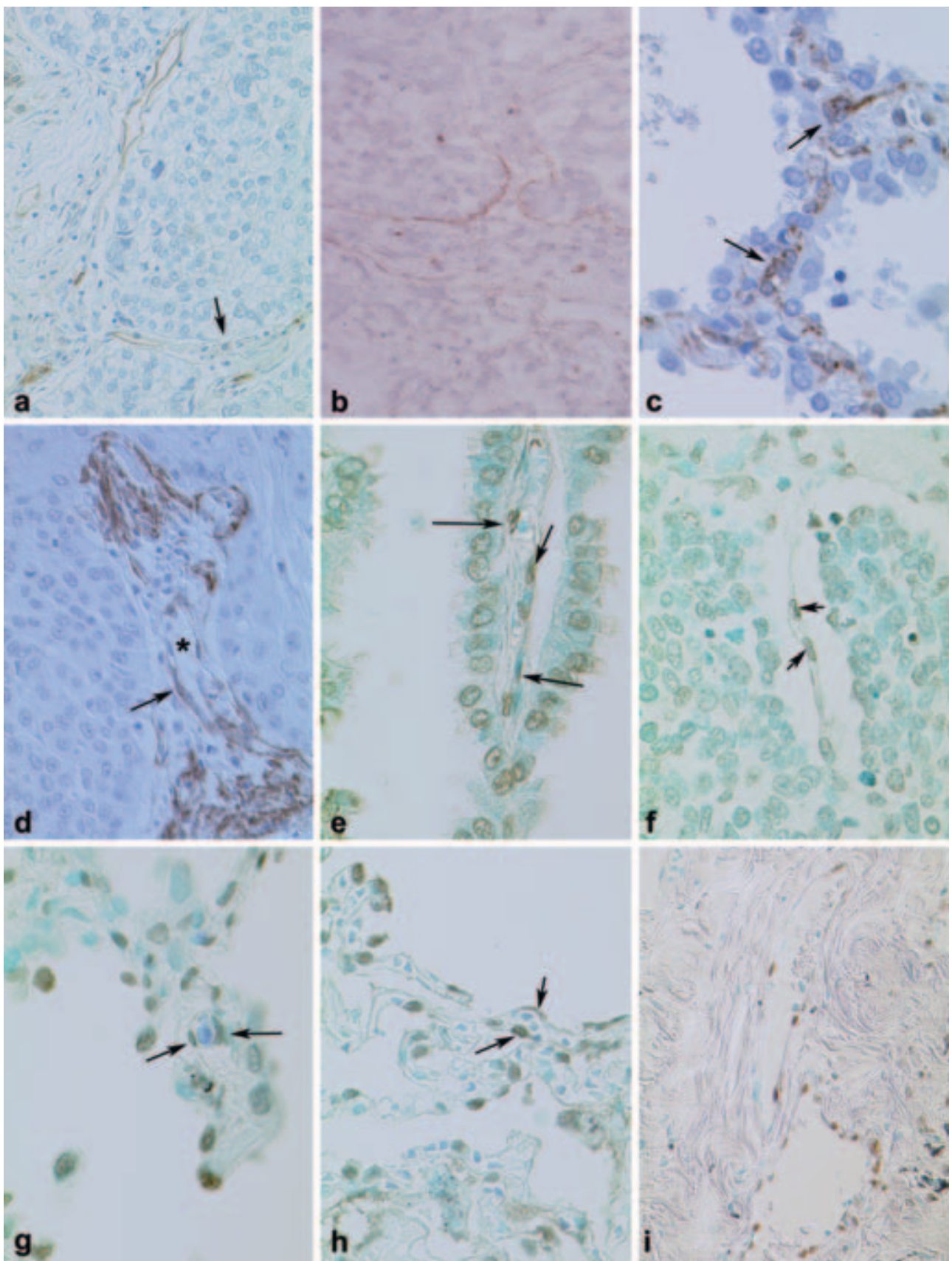

Fig. 2

a) In deeper areas of SCC, parts of alveolar capillaries showed an occasional loss of CD31 expression (arrow). (Immunoperoxidase reaction, $\times 400$ )

b) Patchy lines of type VII collagen were present in the remnants of normal basement membrane layer in the airways. Neoplastic cells did not develop type VII collagen along the alveolar walls in ADC or SCC. (Immunoperoxidase reaction, $\times 400$ )

c) In ADC, pericytes/myofibroblasts often extended cytoplasmic processes containing $\alpha$-SMA-positive intermediate filaments along the interstitial matrix. The nuclei of these cells (arrows) were often hypertrophic and contained large nucleoli, as found in endothelial cells undergoing angiogenic switching. (Immunoperoxidase reaction, $\times 400$ )

d) In SCC, capillaries showed irregular dilatation (asterisk), and the cytoplasm of endothelial cells and pericapillary pericytes (arrow) was attenuated. The pericapillary interstitium was occasionally enlarged by the accumulation of $\alpha$-SMA rich myofibroblasts. (Immunoperoxidase reaction, $\times 400$ )

e-f) Alveolar capillary endothelial cells and neoplastic cells in the alveolar sacs were frequently positive for TUNEL in both ADC (Fig. 2-e, arrows) and SCC (Fig. 2-f, arrows). (TUNEL assay, × 400)

g) TUNEL-positive capillary endothelial cells (arrows) were more frequently found at the normal border of the primary ADC. (TUNEL assay, $\times 400)$

h-i) At the normal borders of ADC (h) and SCC (i), capillary endothelial cells often underwent apoptosis (arrows) together with type II alveolar epithelial cells in the peripheral lung. (TUNEL assay, $\times 200$ ) 
Table 1 Staining properties of cells and basement membrane in alveolar walls

\begin{tabular}{lccc}
\hline & Normal & ADC & SCC \\
\hline Capillary endothelial cells & & & \\
CD31 & + & + & - \\
Thrombomodulin & + & - & - \\
vWF & - & + & - \\
Pericytes/Fibroblasts & & & \\
$\quad \alpha$-SMA & - & + & $+*$ \\
BM (basement membrane) & & & \\
Type IV collagen & + & + & - \\
Type VII collagen & - & - & - \\
\hline$+*$ : no to various amounts of $\alpha$-SMA & &
\end{tabular}

attenuated, as demonstrated with CD31 staining (Fig. 2-a) (Table 1).

The TUNEL assay demonstrated that endothelial cells of alveolar capillaries, as well as neoplastic cells, were frequently positive in both $\mathrm{ADC}$ (Fig. 2-e, arrows) and SCC (Fig. 2-f, arrows). A frequency similar to that shown with the TUNEL assay was seen in caspase 3 (active form) staining in both ADC and SCC (data not shown). Staining with TUNEL was found in an average of $44.9 \%$ (28.9\% to $63.3 \%)$ of the capillary endothelial cells at the frontier zone compared with an average of $34.3 \%$ (32.0\% to $36.5 \%$ ) of those in the deeper portion of the primary $\mathrm{ADC}$ $(P<0.03$; Fig. 2-g, arrows). In SCC, an average of $63.0 \% \quad(46.8 \%$ to $93.9 \%)$ of alveolar capillary counterparts were TUNEL-positive at the tumor periphery, and an average of $53.7 \%$ (40.0\% to $65.3 \%)$ were TUNEL-positive in the deeper portion of the primary tumor mass (Fig. 2-h; $P<0.36$, arrows). The percentage of TUNEL-positive capillary or microvessel endothelial cells (Fig. 2-i) was significantly higher at the peripheral borders in ADC than in SCC $(P<0.02)$. In deeper zones, however, the frequency of TUNEL-positive capillaries was not significantly different between ADC and SCC $(P<0.11)$. Immunohistological characteristics of alveolar capillaries are listed in Table 1.

\section{RT-PCR Analysis of VEGF and VEGFR mRNA}

\section{Expression}

RT-PCR analysis demonstrated that mRNA levels of all isoforms of VEGF were readily detectable from

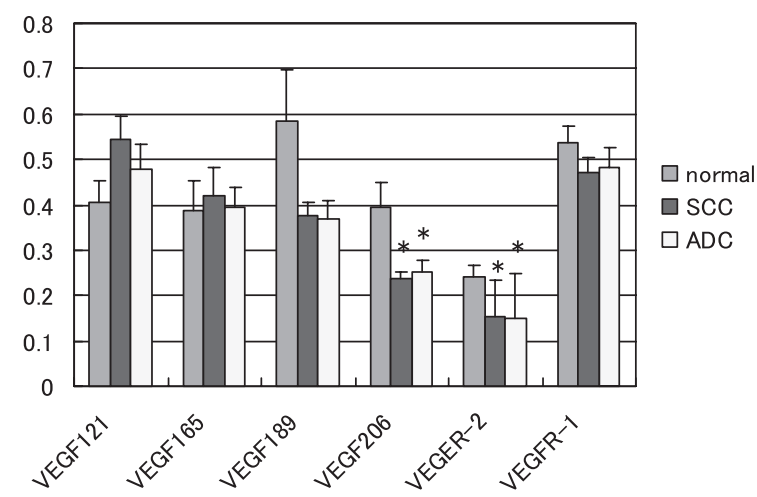

Fig. 3 Expression of mRNA for VEGF isoforms, VEGFR-1, and VEGFR-2 in alveolar wall tissues microdissected with laser capture. The mRNAs expression patterns of VEGFR1 and VEGF isoforms, including VEGF 121 , $\mathrm{VEGF}_{165}$, and $\mathrm{VEGF}_{189}$, did not differ significantly between the control and the cancer groups. The expression of VEGF206 and VEGFR-2 mRNAs was significantly lower in both $\mathrm{ADC}$ and SCC than in the normal lung, but there were no differences between ADC and SCC.

alveolar wall tissues of normal controls, ADC and SCC (Fig. 3). The expression pattern of mRNAs for VEGFR-1 and the VEGF isoforms, including most critical angiogenic factors like $\mathrm{VEGF}_{121}, \mathrm{VEGF}_{165}$, and $\mathrm{VEGF}_{189}$, were not significantly different between the control and the cancer groups. The expression levels of $\mathrm{VEGF}_{206}$ and VEGFR-2 mRNA in the alveolar structures were lower in both ADC and SCC than in the normal lung, but there were no differences between ADC and SCC.

\section{Discussion}

Primary lung ADC occurs frequently at the periphery of the lungs in nonsmokers. The neoplastic cells are thought to originate from glandular, mucous-producing, type II alveolar epithelial, and/or non-ciliated small airway epithelial cells. Tumor cells in bronchioloalveolar carcinoma or papillary ADC tend to show a stepwise spreading on the surface of alveolar walls and gradual expansion into adjacent normal lung structures. In contrast, epithelial cells of the proximal airways easily undergo squamous metaplasia in heavy smokers and sometimes undergo malignant transformation. The 
SCC lesions often appear to contain microscopic necrotic foci. Large necrotic cavities are also present in many cases of SCC and are presumably due to tumor growth exceeding the rate of neovascularization and to narrowing of the vascular lumen by obliterative intimal fibrosis ${ }^{17}$.

To determine whether angiogenic switching occurs in alveolar capillaries in non-small cell lung carcinoma, we characterized the immunohistochemical phenotypes of endothelial cells and the adjacent mesenchymal cells. We performed RT-PCR analysis of VEGF/VEGFR mRNA expression in the microenvironments of the alveolar walls of ADC and SCC. The normal alveolar capillary endothelium was quiescent, as shown by expression of CD31 and the anticoagulant glycoprotein thrombomodulin on its surfaces, and vWF was scarcely present in the cytoplasm (Table 1). In primary lung ADC, alveolar capillaries showed de novo transformation to tumor vessels, but such transformation was not observed in SCC.

At the normal-appearing border area of ADC lesions, endothelial cells showed vWF expression in a few cytoplasmic segments, whereas the surfaces of the endothelium lost thrombomodulin expression in cytoplasm as a segmental unit of cells. The cytoplasmic expression of vWF occurred in whole segments of endothelial cells located below neoplastic cells, whereas CD31 expression remained as in normal capillaries. These findings agree with those of our previous report ${ }^{2,12,13}$, which also demonstrated the development of ultrastructural fenestrae in capillary endothelial cells. The formation of fenestrated capillaries is considered to indicate VEGF-induced production of immature tumor vessels. The present study clearly showed that angiogenic switching occurs in normal alveolar capillary endothelium in primary lung ADC. The capillary pericytes and interstitial fibroblasts simultaneously became hypertrophic and accumulated a large amount of $\alpha$-SMA in the cytoplasm. Alveolar walls frequently showed collagen deposition in the interstitial matrix. Hypoxia due to alveolar fibrosis is not of primary importance for angiogenic switching in this case, because the tumor vessels were apparently formed in the alveolar walls, which were highly attenuated in bronchioloalveolar carcinoma and papillary ADC.

In SCC, the tumor expanded like a compact balloon into alveolar spaces. Pre-existing alveolar wall tissues underwent degenerative changes, as shown by H\&E staining. This degeneration was confirmed by the irregular or total loss of staining for thrombomodulin, vWF, and CD31 in the capillary endothelial cells. As a consequence, capillary lumens were dilated, the cytoplasm of endothelial cells was irregularly attenuated, and the nuclei were often pyknotic, suggesting degeneration or necrosis in alveolar capillaries.

Staining for type IV collagen in the SCC often became faint along the capillary sheath. No positive staining was found for type VII collagen along the neoplastic cells of the SCC or ADC. Only remnants of the collagen were found along normal airways in cancerous lesions. Our previous studies demonstrated type VII collagen by immunohistochemistry and electron microscopy as anchoring fibrils, one of the major adhesive components in the basement membrane zone, in normal and fibrotic lungs ${ }^{16,18-22}$. Catusse et $a l^{23}$ have examined type IV and type VII collagens at different stages of bronchopulmonary cancers. In well-differentiated carcinomas, type VII collagen was detected in newly synthetized basement membrane. In contrast, poorly differentiated invasive cancers did not deposit type VII collagen, whereas the $\alpha 1$ chain of type IV collagen persisted. They claimed that the most important modifications in basement membrane composition during tumor progression was the appearance of the $\alpha 3$ chain of type IV collagen in well-differentiated carcinomas and its subsequent disappearance in poorly differentiated carcinomas, along with the consistent loss of type VII collagen. However, they did not clearly define where the cancer cells were located in relation to alveolar walls and whether they were classified according to histological types. Wetzels et al. ${ }^{24-27}$, in contrast, have reported that type VII collagen is present only in SCC and that its expression is reduced with decreasing differentiation, whereas type VII collagen was not identified in ADC, small cell carcinomas, or carcinoids. Our present results 
show that type VII collagen is absent in SCC and ADC. The discrepancy between these reports might be due, in part, to the antibodies or methods used or the areas examined, as our observation was focused on the extending border area of neoplastic cells.

We found that the percentage of TUNEL-positive cells was significantly higher in alveolar capillaries than in the deeper zone of primary lung ADC $(P<$ 0.03). Tumor vessels are considered dynamic, immature, and destabilized, which allows sprouting at the extending edge of the tumor ${ }^{5}$. The reaction might cause the cells to be vulnerable to apoptosis. Apoptosis was more frequent in ADC than in SCC, whereas VEGF and VEGFR-2 mRNA expression patterns did not have difference between the two types of cancer. This result agrees with the observation that ADC shows a greater sensitivity to apoptosis than does $\mathrm{SCC}^{28}$, corresponding to ADC's higher need for angiogenesis and poorer prognosis. Jin et al. ${ }^{11}$ have reported that the levels of mRNAs encoding soluble isoforms of VEGF and VEGFR-2 in alveolar walls are much higher in primary lung ADC than in normal lungs. These results suggest that diffusible isoforms of VEGF released from neoplastic cells play a key role in the induction of cell growth in alveolar capillary endothelial cells. Despite similar patterns of VEGF and VEGFR mRNA expression in $\mathrm{ADC}$ and SCC, the poor angiogenesis or insufficient maintenance of alveolar capillaries in SCC might be due to the lack of close contact between neoplastic cells and the underlying interstitial matrix. The poor epithelial-mesenchymal contact in SCC demonstrated in this study is in sharp contrast to that in ADC. Extensive lack of nourishment for neoplastic cells in SCC might depend on an insufficient blood supply.

The angiogenic force is derived from a large series of growth factors ${ }^{1-6}$, which are controlled by the activities of their receptors ${ }^{7-10}$ or cofactors $^{14}$ or both. The expression of VEGF-related mRNAs depend upon cell types involved in alveolar wall tissues microdissected from ADC and SCC. Occasional increases in the number of fibroblasts were found in both types of lung cancer, which does not substantially cause expression of mRNA of VEGF-VEGFR. Thus, the result of RT-PCR in the present study could not be interfered by types of cells other than neoplastic cells and endothelial cells in alveolar walls.

Tumor angiogenesis might be suppressed by other process as suggested by Padera et al. ${ }^{29}$ They have provided evidence that proliferating cancer cells cause intratumor vessels to compress, leading to the collapse of capillaries. With the overpressure of the expanding tumor mass of SCC, the narrowing of capillary lumens could lead to insufficient oxygenation of alveolar wall cells and, occasionally, to irregular dilatation at their peripheral branches. Suppressive factors for alveolar capillary angiogenesis should be taken into consideration in the treatment of cancer, especially in early stages of primary SCC of the lung, although the details of factors remain yet unclear at this stage of study.

\section{References}

1. Hanahan D, Folkman J: Patterns and emerging mechanisms of the angiogenic switch during tumorigenesis. Cell 1996; 86: 353-364.

2. Metheny-Barlow LJ, Li LY: The enigmatic role of angiopoietin-1 in tumor angiogenesis. Cell Res 2003; 13: 309-317.

3. Folkman J: Tumor angiogenesis: a possible control point in tumor growth. Ann Intern Med 1975; 82: 96100.

4. Folkman J: What is the evidence that tumors are angiogenesis dependent? J Natl Cancer Inst 1990; 82: 4-6.

5. Yancopoulos GD, Davis S, Gale NW, Rudge JS, Wiegand SJ, Holash J: Vascular-specific growth factors and blood vessel formation. Nature 2000; 407: 242-248.

6. Gale NW, Yancopoulos GD: Growth factors acting via endothelial cell-specific receptor tyrosine kinases: VEGFs, angiopoietins, and ephrins in vascular development. Genes Dev 1999; 13: 1055-1066.

7. Suri C, Jones PF, Patan S, et al.: Requisite role of angiopoietin-1, a ligand for the TIE2 receptor, during embryonic angiogenesis. Cell 1996; 87: 1171-1180.

8. Maisonpierre PC, Suri C, Jones PF, et al: Angiopoietin-2, a natural antagonist for Tie2 that disrupts in vivo angiogenesis. Science 1997; 277: 5560.

9. Holash J, Wiegand SJ, Yancopoulos GD: New model of tumor angiogenesis: dynamic balance between vessel regression and growth mediated by angiopoietins and VEGF. Oncogene 1999; 18: 53565362.

10. Holash J, Maisonpierre PC, Compton D, et al: Vessel cooption, regression, and growth in tumors mediated by angiopoietins and VEGF. Science 1999; 284: 19941998.

11. Jin E, Ghazizadeh $M$, Fujiwara $M$, et al: Angiogenesis and phenotypic alteration of alveolar 
capillary endothelium in areas of neoplastic cell spread in primary lung adenocarcinoma. Pathol Int 2001; 51: 691-700.

12. Jin E, Fujiwara M, Nagashima M, et al.: Aerogenous spread of primary lung adenocarcinoma induces ultrastructural remodeling of the alveolar capillary endothelium. Hum Pathol 2001; 32: 1050-1058.

13. Kawanami O, Jin E, Ghazizadeh M, et al.: Mosaic-like distribution of endothelial cell antigens in capillaries and juxta-alveolar microvessels in the normal human lung. Pathol Int 2000; 50: 136-141.

14. Jin E, Fujiwara M, Pan X, et al: Protease-activated receptor (PAR)-1 and PAR-2 participate in the cell growth of alveolar capillary endothelium in primary lung adenocarcinomas. Cancer 2003; 97: 703-713.

15. Koukourakis MI, Giatromanolaki A, Thorpe PE, et al: Vascular endothelial growth factor/KDR activated microvessel density versus CD31 standard microvessel density in non-small cell lung cancer. Cancer Res 2000; 60: 3088-3095.

16. Nakajima M, Kawanami O, Jin E, et al. Immunohistochemical and ultrastructural studies of basal cells, Clara cells and bronchiolar cuboidal cells in normal human airways. Pathol Int 1998; 48: 944953.

17. Hasleton PS: Spencer's Pathology of the Lung Fifth edition, 1996; pp 1020-1064, McGraw-Hill Companies, New York.

18. Kawanami O, Ferrans VJ, Crystal RG: Anchoring fibris in the normal canine respiratory system. Am Rev Respir Dis 1979; 120: 595-611.

19. Kawanami O, Ferrans VJ, Roberts WC, Crystal RG, Fulmer JD: Anchoring fibris: A new connective tissue structure in fibrotic lung disease. Am J Pathol 1978; 92: 389-410.

20. Kawanami O, Basset F, Ferrams VJ, Soler P, Crystal RG: Plumonary Langerhans' cells in patients with fibrotic lung disorders. Lab Invest 1981; 44: 227-233.

21. Kawanami O, Ferrans VJ, Crystal RG: Structure of alveolar epithelial cells in patients with fibrotic lung disorders. Lab Invest 1982; 46: 39-53.
22. Kawanami O: Ultrastructure of the lung in health and in fibrotic diseases. In Basic and Clinical Aspects of Plumonary Fibrosis (Takishima T, ed), 1994; pp 29-73, CRC Press, Boca Raton.

23. Catusse C, Polette M, Coraux C, Burlet H, Birembaut $\mathrm{P}$ : Modified basement membrane composition during bronchopulmonary tumor progression. J Histochem Cytochem 2000; 48: 663-669.

24. Wetzels RH, van der Velden LA, Schaafsma HE, et al.: Immunohistochemical localization of basement membrane type VII collagen and laminin in neoplasms of the head and neck. Histopathology 1992; 21: 459-464.

25. Wetzels RH, Robben HC, Leigh IM, Schaafsma HE, Vooijs GP, Ramaekers FC: Distribution patterns of type VII collagen in normal and malignant human tissues. Am J Pathol 1991; 139: 451-459.

26. Wetzels RH, Schaafsma HE, Leigh IM, et al.: Laminin and type VII collagen distribution in different types of human lung carcinoma:correlation with expression of keratins 14, 16, 17 and 18. Histopathology 1992; 20: 295-303.

27. Wetzels RH, Holland R, van Haelst UJ, Lane EB, Leigh IM, Ramaekers FC: Detection of basement membrane components and basal cell keratin 14 in noninvasive and invasive carcinomas of the breast. Am J Pathol 1989; 134: 571-579.

28. Nakashima T, Huang CL, Liu D, et al.: Expression of vascular endothelial growth factor-A and vascular endothelial growth factor-C as prognostic factors for non-small cell lung cancer. Med Sci Monit 2004; 10: $157-165$.

29. Padera TP, Stoll BR, Tooredman JB, Capen D, di Tomaso E, Jain RK: Pathology: cancer cells compress intratumour vessels. Nature 2004; 427: 695.

(Received, March 1, 2007)

(Accepted, July 4, 2007) 